\title{
Ein Land, nur Opfer? Zu Gruppenstereotypen in der Auseinan- dersetzung um die lettische Sprachenpolitik am Beispiel des Sprachreferendums von 2012
}

\author{
Sandra J. Langer (Riga)
}

\begin{abstract}
On February 18th 2012 the referendum on the Draft Law 'Amendments to the Constitution of the Republic of Latvia' took place in Latvia. Its purpose was to decide whether or not the Russian language should be made the second official language of the Republic of Latvia. But this referendum was just the preliminary climax in a chain of events that exposed the relations between the ethnic Latvian and the ethnic Russian parts of Latvian society. The burden of historical events concerning the loss of Latvia's independence after World War II and its assimilation in the Sowjet Union including deportations and the repression of the Latvian national identity still effects the relations between Latvia's citizens, especially when it comes to political decisions. This article gives an overview over the chain of events leading to the referendum including observations about the perticular importance of language in terms of national identity. The main focus, however, will be on the analysis of relations between ethnic Latvians and ethnic Russians based on Niklas Luhmann's concept of trust. Mistrust as the downside of trust is constitutive for many parts of Latvian public life, which is widely discussed in all social sectors and even passed on through the generations. Considering the trust issues within Latvian society severe consequences for everyday life can be detected: longterm stereotypes and prejudice between the ethnic groups. In this article the kind and content of the stereotypes and prejudices are named and analyzed. This leads to the question, if it is even necessary to use Erving Goffmans concept of stigma to describe the relations.
\end{abstract}

\section{$1 \quad$ Einleitung}

Deutschland und Dänemark sind durch klare Staatsgrenzen territorial und politisch voneinander getrennt. Im deutsch-dänischen Grenzgebiet kommt es zu Begegnungen und zum gegenseitigen Austausch, aber die politischen Machtverhältnisse und Zuständigkeiten bleiben klar geregelt. Stereotype über die jeweils andere Gruppe sind daher strukturell und funktional eingebunden in ein Selbstverständnis, das von dem Selbstbewusstsein der Eigenstaatlichkeit getragen ist. Völlig anders verhält es sich in Lettland. Der ethnisch lettische und der ethnisch russische Teil der Bevölkerung leben gemeinsam innerhalb der lettischen Staatsgrenzen und eine einvernehmliche Einrichtung des Gemeinwesens wird erschwert durch das Empfinden fehlender Gemeinsamkeit, das Ringen um politische Eindeutigkeit und die unterschiedliche

Linguistik online 79, 5/2016 - http://dx.doi.org/10.13092/lo.79.3336

CC by 3.0 
Auslegung der Vergangenheit, das Herausbilden einer gemeinsamen Gruppenidentität ist mittelfristig nicht abzusehen. Anhand eines konkreten Beispiels, dem großen Sprachreferendum vom 18. Februar 2012, sollen die Deutungsmuster herausgearbeitet werden, die eine Annäherung der beiden Bevölkerungsteile erschweren oder gar verhindern.

Ziel des Referendums war, die Verfassung zu ändern und Russisch zur zweiten Amtssprache Lettlands zu machen. Dieser Versuch, die russische Sprache in den Strukturen des lettischen Staates zu verankern führte zwischen dem Sommer 2011 und Frühjahr 2012 in Lettland zu einer breiten öffentlichen Diskussion und Medienaufmerksamkeit (cf. Bongartz 2012). Das Verhältnis beider Bevölkerungsgruppen ist durch die politischen Entwicklungen des 20. Jahrhunderts belastet und die Frage nach einer zweiten Amtssprache weckte auf beiden Seiten unterschiedliche Befürchtungen oder gar Verärgerung und führte zu einer allgemeinen Verunsicherung. Die Beteiligung am Referendum lag mit 70,66 Prozent oder 1.091.757 Wahlbürgern höher als bei Parlamentswahlen. Die Frage der Gruppenzugehörigkeit und Identitätsbildung ist auch in Lettland in hohem Maße an den Sprachgebrauch gebunden. „In addition to phenotype, each community has a repertoire of language ideologies that come into play in the ascription of ethnicity." (Fought 2006: 31)1 Entsprechend grenzen sich der Ethnisch lettische und der ethnisch russische Bevölkerungsteil auch durch Sprache deutlich erkennbar voneinander ab. Eine Verweigerung der lettischen Sprache von Seiten der ethnischen Russen wird jedoch von den ethnischen Letten gleichgesetzt mit einer Verweigerung gegenüber dem Staat Lettland und dem Gemeinwesen Lettlands. Entsprechend verfügt das Land seit der ersten Staatsgründung 1918 über eine Reihe von Sprachgesetzen, die den Status des Lettischen und seine Anwendungsgebiete genau festlegen. Mit der wiedererlangten staatlichen Unabhängigkeit kam es zum Sprachgesetz von 1989/1992, welches wiederum 1999 vom lettischen Parlament (Saeima) ersetzt wurde. Das aktuelle Sprachgesetz trat am 1. September 2000 in Kraft (cf. Vvl s. a.). Artikel fünf des Sprachgesetzes legt fest: Jegliche weitere in der Lettischen Republik verwendete Sprache, ausgenommen das Livische, wird nach dem Verständnis dieses Gesetzes als Fremdsprache angesehen (cf. Vvl s. a.). ${ }^{2}$ Eine Differenzierung in Minderheitensprachen oder Regionalsprachen fehlt. Das Russische hat nach diesem Gesetz den Status einer Fremdsprache in Lettland. Ebenso legt die Verfassung Lettlands in Artikel vier (in Kraft getreten 1998) fest: Die Staatssprache der Republik Lettland ist die lettische Sprache (cf. LRS s. a.). ${ }^{3}$ Bereits in diesen restriktiven Sprachregelungen Lettlands zeigt sich eine grundsätzliche Tendenz:

„Sanctions on the refusal to use a code are particularly prominent in bilingual communities“ (Fought 2006: 29). Im Gegenzug kann aber die Sprache auch als entscheidendes Verbindungselement fungieren:

\footnotetext{
${ }^{1}$ Die besondere Rolle der Kategorie der Ethnizität offenbart sich für die vorliegende Untersuchung darin, dass in den Pässen der Sowjetunion die Ethnizität als eigene Kategorie angegeben war. Später herrschte in Lettland eine Diskussion darüber, ob diese Klassifizierung offiziell abzuschaffen sei, die Meinungen darüber bleiben gespalten.

${ }^{2}$ Im Original: „Ikviena cita Latvijas Republikā lietotā valoda, izṇemot lībiešu valodu, šā likuma izpratnē ir uzskatāma par svešvalodu.“ [Übersetzung SJL]

${ }^{3}$ Im Original: „Valsts valoda Latvijas Republikā ir latviešu valoda.“ [Übersetzung SJL]
}

ISSN 1615-3014 
As Buchholz puts it, "the ideological link between language and ethnicity is so potent that the use of linguistic practices associated with a given ethnic group may be sufficient for an individual to pass as a group member".

(Fought 2006: 32)

Erste Analysen zum öffentlichen Diskurs mit Bezug auf das Referendum wurden am 27./28. November 2014 im Rahmen der Tagung „, Verfall der Sitten und Moral“ - Diskursanalysen der Ablehnung/Abgrenzung gesellschaftlicher Wertvorstellungen in Riga sowohl von Jūlija Zālīte, als auch von Sandra J. Langer präsentiert. Die vorliegende Untersuchung stellt eine Weiterführung dar, in der zunächst die Umstände des Referendums umrissen werden, um anschließend unter Zuhilfenahme der kulturphilosophischen Kategorie des Vertrauens bzw. Misstrauens die Grundproblematik zu erörtern. Daraufhin wird unter kulturpsychologischer Perspektive gezielt nach der Genese und Funktion von Stereotypen zwischen ethnisch lettischen und ethnisch russischen Einwohnern gefragt sowie nach den Mechanismen ihrer Aufrechterhaltung. Damit verbunden ist auch die Frage, ob die wechselseitigen Wahrnehmungsmuster, die sich verschiedentlich im Alltagsdiskurs, in konkreten Handlungen oder gelebter Distanzierung ausdrücken, tatsächlich als Stereotype aufzufassen sind, denn abschließend wird die Perspektive noch kontrastiv um die Kategorie des Stigmas nach Goffman erweitert. Die Frage nach den Aspekten der Deutungsmuster, die die Genese einer gemeinsamen Gruppenidentität für ethnisch lettische und ethnisch russische Personen erschweren oder gar verhindern, soll anhand weniger, markanter Beispieläußerungen abgeleitet verdeutlicht werden. Diese Äußerungen beinhalten, wie noch gezeigt wird, auch die Unterstellung des Versuchs einer politischen Destabilisierung Lettlands durch Einflüsse aus Russland. Die Frage nach einer tatsächlichen versuchten Einflussnahme muss an dieser Stelle offen bleiben. Relevant für die vorliegenden Überlegungen ist vielmehr die Frage nach dem Deutungsmuster, unabhängig von seinem Wahrheitsgehalt.

\section{Phasen des Referendums und historische Voraussetzungen}

Ausgangspunkt des Referendums war die vom Privatmann ${ }^{4}$ Vladimirs Lindermans initiierte Gründung der Initiative Dzimtā Valoda (Muttersprache). Nach ersten Bemühungen für die russische Sprache überreichte Lindermans der Zentralen Wahlkommission Lettlands im Sommer 2011 eine Unterschriftenliste mit 12.533 Unterschriften, wodurch die Kommission gesetzlich verpflichtet war, innerhalb Lettlands und in lettischen Botschaften im Ausland eine erste Abstimmung zu ermöglichen. Bei diesem ersten Referendum im November 2011 ging es bereits um die Frage nach Russisch als zweiter Amtssprache in Lettland, allerdings galt es hier nur abzustimmen, sofern man die Verfassungsänderung unterstützte. Das zu erreichende Quorum lag bei mindestens einem Zehntel der Bevölkerung bzw. 154.379 stimmberechtigten Bürgern. (cf. Bongartz 2011a; Bongartz 2011b). Dies ist insofern beachtenswert, als in Lettland 1.817.866 Personen mit lettischer Staatsbürgerschaft gemeldet sind, daneben aber 276.797 sogenannte Nichtbürger russischer Ethnie und 51.565 russische Staatsbürger. ${ }^{5}$ Die Nichtbürger gelten zwar als dem Staat zugehörig, sind also nicht zu verwechseln mit den

\footnotetext{
${ }^{4}$ Es handelt sich explizit nicht um die Initiative einer bestehenden Interessensvertretung der ethnischen Russen oder einer politischen Partei, obwohl es hier ein Spektrum an Sympathisanten gegeben hätte. Es bleibt zu spekulieren, ob der Anstoß als genuin aus dem Volk heraus kommend artikuliert oder inszeniert wurde.

${ }^{5}$ Angaben des Lettisches Migrationsamts, Stand 1. Juli 2014.
} 
Staatenlosen, sie sind jedoch nicht wahlberechtigt. Bei diesem ersten Referendum wurde das Quorum erreicht (183.046 Bürger stimmten für die zweite Amtssprache). Daraufhin war Staatspräsident Andris Bērziņš verpflichtet den artikulierten Wunsch nach einer Verfassungsänderung den Saeima-Abgeordneten vorzulegen. Der Antrag auf Verfassungsänderung hätte dann mit einer Zweidrittel-Mehrheit verabschiedet werden müssen, andernfalls würde eine allgemeine Volksabstimmung nötig (cf. Bongartz 2011b). Am 22. Dezember 2011 lehnte die Saeima eine Verfassungsänderung ab. „60 Parlamentarier lehnten Russisch als Amtssprache ab. Niemand stimmte dafür" (Bongartz 2011c), eine Reihe von Abgeordneten enthielt sich der Stimme. Die allgemeine Volksabstimmung, die als das eigentliche lettische Sprachreferendum 2012 wahrgenommen wurde, musste nun erfolgen. Die Bekanntmachung der Rahmenbedingungen seitens der Zentralen Wahlkommission Lettlands lautete:

On Saturday, 18 February 2012 the referendum on the "Draft Law Amendments to the Constitution of the Republic of Latvia" was held in Latvia. The Draft law provided for the amendments of Article 4, 18, 21, 101 and 104 of the Constitution by adding the condition about Russian as the second official language, prescribing two working languages - Latvian and Russian for selfgovernment institutions, as well as establishing the rights for everyone to receive information in Latvian and Russian. The ballot papers contained the question "Do you support the adoption of the Draft Law 'Amendments to the Constitution of the Republic of Latvia' that provides for the Russian language the status of the second official language". There were two possible answers "For" and "Against".

(CVL 2012)

So waren an dieser Phase alle stimmberechtigten Bürger aufgerufen ihre Unterstützung oder Ablehnung zu dokumentieren. Abermals waren die Nichtbürger strukturell von der Abstimmung ausgeschlossen. Die zentrale Wahlkommission fasst die Ergebnisse der Abstimmung folgendermaßen zusammen:

The referendum results summarized by the Central Election Commission show that 273,347 voters cast their votes for adopting of the amendments to the Constitution, while 821,722 voters were against the amendments to the Constitution. 3,524 or $0.32 \%$ ballots were recognized as invalid since voters matched the both answers "For" and "Against" or no one of these answers was matched on these ballot papers. There were 1,098,921 citizens registered in voters' lists of polling stations as voters participated in voting. Article 79 of the Constitution of the Republic of Latvia stipulates that amendments to the Constitution submitted to a referendum, shall be adopted, if at least one-half of those who have the right to vote have declared themselves in their favour. According to information from the Population Register of the Office of Citizenship and Migration Affairs there were 1,545,004 citizens of Latvia having the right to vote on 18 February 2012. Therefore the results of the referendum show that the amendments to the Constitutions were not supported since the number of votes "For" received in the referendum was less then 772,502 or one-half of voters who have the right to vote.

(CVL 2012)

Bei dem Referendum handelte es sich um einen legalen demokratischen Akt, die vorausgehende Entwicklung schöpfte die Möglichkeiten bürgerlicher Mitbestimmung in Lettland aus, wobei das Anliegen letztlich keine Mehrheit fand und nicht umgesetzt wurde. Am Diskurs um das Referendum offenbart sich jedoch ein grundsätzlicher Konflikt. Udo Bongartz erwähnt, dass das Referendum möglicherweise nur als eine Reaktion auf die nationalkonservativen 
Politiker in Lettland zu werten sei, die zuvor die Finanzierung russischer Schulen durch den Staat beenden wollten. „Aber das Referendum [bezüglich der Schulen] scheiterte wegen mangelnder Teilnahme. Linderman begründete seine Russisch-Initiative als Reaktion auf den Vorstoß der lettischen Nationalisten“ (Bongartz 2011b). Ob dies der tatsächliche oder ein vorgeschobener Grund ist, wird an dieser Stelle nicht zu klären sein. Vielmehr zeigt sich aber schon an der Spekulation um die Hintergründe des Referendums ein Konflikt um die gesellschaftliche sowie politische Selbst- und Mitbestimmung der beiden großen ethnischen Gruppen des Landes.

Das heutige Territorium Lettlands wurde vom 13. bis ins 18. Jahrhundert von immer wieder wechselnden Herrschern regiert, wobei die dort ansässigen Deutschen bis ins 19. Jahrhundert die gesellschaftliche Oberschicht gebildet haben. Im 18. Jahrhundert wurde das Gebiet politisch an das russische Zarenreich angegliedert und es kam zu einer ersten Russifizierung, als die russische Sprache im ausgehenden 19. Jahrhundert zur Unterrichtssprache in den Bildungsinstitutionen wurde und hier das Deutsche ablöste. Ebenfalls in der zweiten Hälfte des 19. Jahrhunderts begann das nationale Erwachen der Letten, die zunehmend einen eigenen Nationalstaat einforderten. Doch obgleich Lettland bereits im 19. Jahrhundert Teil des russischen Zarenreichs war, geht die Geschichte der aktuellen lettisch-russischen Spannungen im Wesentlichen auf das 20. Jahrhundert zurück. Die staatliche Eigenständigkeit der Republik Lettland, die am 18. November 1918 proklamiert wurde, endete mit der Angliederung der Lettischen SSR an die Sowjetunion in den 40er Jahren des 20. Jahrhunderts. In dieser Phase, bis zur wiedererlangten Unabhängigkeit am 4. Mai 1990, wurzelt der Konflikt zwischen den Bevölkerungsteilen. Die Erfahrungen innerhalb der Sowjetunion, Massendeportationen sowie eine aggressive Russifizierungspolitik haben die ethnisch lettische Bevölkerung des heutigen Lettlands nachhaltig geprägt. Die Russifizierung beinhaltete unter anderem zwei markante Aspekte: die Unterdrückung der lettischen Sprache und Kultur zugunsten der russischen sowie die gezielte Ansiedlung russischer Bürger auf lettischem Gebiet. „Vor 1939 hatte die russische Minderheit gerade einmal neun Prozent der Einwohner betragen. Heute sind ein Drittel der Bevölkerung Russen“ (Gnauck 2012).

\section{Das Referendum unter dem Gesichtspunkt von Vertrauen}

Das heutige Verhältnis der Bevölkerungsgruppen zueinander lässt sich unter dem von Niklas Luhmann näher dargestellten Gesichtspunkt des Vertrauens bzw. Misstrauens fassen. Nach Luhmann ist Vertrauen eine in die Zukunft gerichtete Notwendigkeit der Komplexitätsreduktion. Misstrauen ist jedoch nicht das Gegenteil, sondern ,ein funktionales Äquivalent für Vertrauen“ (2000: 93), das unter anderen Vorzeichen' ${ }^{6}$ gleichfalls der Komplexitätsreduktion dient. Beide sind ,symbolisch vermittelte, generalisierte Haltungen [...], die nicht mit spezifisch angebbaren objektiven Ursachen variieren, sondern durch subjektive Prozesse der vereinfachenden Erlebnisverarbeitung gesteuert werden“ (Luhmann 2000: 99).

Das Vorhandensein von Misstrauen zeigt sich in den Reaktionen auf das Referendum besonders deutlich. So etwa auch in der Reaktion von Valdis Zatlers, dem ehemaligen Ministerprä-

\footnotetext{
${ }^{6}$,Diese negativen Strategien geben dem Mißtrauen jenes emotional gespannte, oft krampfhafte Naturell, das es vom Vertrauen unterscheidet“" (Luhmann 2000: 93).
} 
sidenten und Begründer einer Reformpartei, die seit 2011 dem lettischen Parlament angehört, auf das Sprachreferendum 2012. „,Das Referendum ist eine Provokation, es soll die Nation spalten", sagte er im Gespräch" (Gnauck 2012). Gleichsam wird das Misstrauen als Problem meist vom Alltagsleben überlagert und bricht daher besonders deutlich hervor, wenn die Alltagsnormalität durch Ereignisse wie das Referendum ${ }^{7}$ durchbrochen wird. Im Alltag des Zusammenlebens geht „das Bewußtsein des Mißtrauens verloren und die ihm zugeordneten Reduktionsstrategien werden als gewohnte Lebensauffassungen, als Routine verselbständigt“ (Luhmann 2000: 93). Luhmann verweist darauf, dass die Vorgeschichte ${ }^{8}$ den entscheidenden Faktor darstellt, der ,,den Äußerungsstil, die Gefühlstönung und die Hartnäckigkeit des Mißtrauens“ begründet (Luhmann 2000: 95). Die eingangs skizzierte Vorgeschichte, der schmerzliche Verlust der Eigenstaatlichkeit und die damit verbundenen Leidensgeschichten der Bevölkerung ${ }^{9}$ sowie die pauschale Behauptung, das lettische Volk hätte die Zugehörigkeit zur Sowjetunion gewünscht, steht von Beginn der zweiten Republik an zwischen den ethnisch lettischen und den ethnisch russischen Einwohnern Lettlands. Die in Untersuchungen und Zeitzeugenberichten dargestellte Diskrepanz zwischen der ideologisch dominierten Staatsdoktrin und den tatsächlichen Lebensumständen der Bevölkerung in der Sowjetunion war nicht geeignet in der breiten Bevölkerung über die Zirkel überzeugter Kommunisten hinaus ein Vertrauensverhältnis zur Regierung in Moskau zu stiften. Die Russifizierungsmaßnahmen auf dem ehemaligen lettischen Staatsgebiet schürten Ängste vor Überfremdung und völligem Verlust der eigenen Identität. So konnte sich zwischen den Letten und den gezielt von der Regierung angesiedelten Russen ebenfalls kein Vertrauensverhältnis entwickeln.

Das zunächst von lettischer Seite eingebrachte Misstrauen wirkt jedoch auf die Gesamtsituation zurück, denn

[z]wischenmenschliches Verhalten wird nicht nur ad hoc erlebt, sondern auf zugrundeliegende ,Einstellungen“ interpretiert und zur Erwartungsbildung benutzt, und so kann der Mißtrauische, ob er will oder nicht, kaum vermeiden, daß sein Mißtrauen ihm angesehen und zugerechnet wird.

(Luhmann 2000: 97)

Die Konsequenz ist eine Spirale des Misstrauens, in der auch die durchschnittlichen, nicht spezifisch politisch interessierten ethnisch russischen Einwohner Lettlands, die in Lettland angesiedelt wurden, auf das ihnen entgegengebrachte Misstrauen ihrerseits mit Misstrauen den ethnisch lettischen Einwohnern gegenüber reagieren. Katja Wezel erklärt das Misstrauen mit den durch die Besatzung entstandenen und von den lettischen Einwohnern wahrgenommenen Machtverhältnissen:

\footnotetext{
${ }^{7}$ Eine spätere und noch markantere Bruchstelle des Zusammenlebens im Alltag stellt die anhaltende Krise in der Ukraine dar.

8 „Man muß die Systemgeschichte kennen, die Geschichte der Selbstdarstellungen, die Bewährung von Prämissen und Symbolen der Erlebnisverarbeitung, von Angstdefensiven und Vereinfachungsmitteln, und ferner die Biographie der jeweiligen Situation, wenn man abschätzen will, wie stark Vertrauen und Mißtrauen sind, wie stark sie innendeterminiert oder an bestimmte Bezugsobjekte gebunden sind und durch welche kritischen Erfahrungen sie geändert werden können“" (Luhmann 2000: 100).

${ }^{9}$ Die Deportationen lettischer Bürger sind heute bekannt und dokumentiert - beispielsweise im lettischen Okkupationsmuseum in Riga - und darüber hinaus Teil zahlreicher lettischer Familienerinnerungen. (cf. Kusber 2014: 6).
} 
Die russischsprachige Minderheit, insbesondere jene, die im Zuge der sowjetischen Besatzung nach 1944 nach Lettland kamen, fühlt sich kollektiv als ,Besatzer' abgestempelt und ungerecht behandelt, da viele von ihnen als Arbeiter, Ingenieure oder Naturwissenschaftler nach Lettland kamen und nicht als Angehörige einer ,Besatzerarmee'. Diesen Einwanderern war in der Regel gar nicht bewusst, dass sie in ein anderes, ein besetztes Land kamen, denn der Sowjetideologie zufolge waren die baltischen Staaten der UDSSR freiwillig beigetreten.

(Wezel 2008: 214)

Karin Liebhart (2009: 124) spricht gar von der ,implizite[n] Gleichsetzung der russischen nationalen Minderheiten mit den ehemaligen sowjetischen Besatzern" und folgert daraus eine „Ethnisierung historischer politischer Konflikte“. Die Herausbildung der Misstrauensspirale wird von Luhman aus der Perspektive derjenigen Gruppe, die zuerst Objekt des Misstrauens geworden ist, folgendermaßen dargestellt:

Wer sich als Gegenstand solcher Mißtrauensäußerungen sieht, wird kaum geneigt sein, die Perspektive des Mißtrauens auf sich selbst anzuwenden und die Ursache dafür in sich selbst zu suchen. Das Mißtrauen bleibt ihm objektiv unerklärlich, und also rechnet er es dem Mißtrauenden zu. Er wird zunächst vielleicht mit Erläuterungen, mit Nachsicht, dann mit Vorsicht und schließlich selbst mit Mißtrauen antworten, sofern er die Beziehung überhaupt fortsetzt. Er findet sich durch das ihm entgegengebrachte Mißtrauen von moralischen Bindungen entlastet, in die Freiheit eines Handelns nach eigenen Interessen versetzt, sofern er nicht gar das Bedürfnis fühlt, sich für unverdiente Behandlung zu rächen. Und dadurch gibt er dem Mißtrauen nachträgliche Rechtfertigung und weitere Nahrung.

(Luhmann 2000: 97-98)

Die Spirale ist in diesem Sinne selbst bestätigend, denn wer nach den eigenen Interessen handelt, weil er sich von der Gemeinschaft entkoppelt fühlt, bestätigt durch seine Handlungen die bereits von der anderen Seite vermutete Nicht-Zugehörigkeit. Beide Gruppen können in Lettland historische Ereignisse für ihre Perspektive geltend machen, die sich um die Achse der Wiederherstellung der staatlichen Unabhängigkeit drehen. Aus der Perspektive der ethnisch lettischen endet hier die erlittene Benachteiligung, aus der Perspektive der ethnisch russischen ist es der Ausgangspunkt ihrer Benachteiligung. Den zugrunde liegenden Dreh- und Angelpunkt thematisierte Carmen Fought als ,the imposition of hierarchical rankings on ethnic groups by the dominant ideology“. Diese Rangordnung ist eine Setzung: ,[u]sually the dominant group is positioned as superior in the ideology" (2006: 15). In der Sowjetunion führt dies zur Hochschätzung des Russischen und Unterdrückung des Lettischen, in der Lettischen Republik hingegen kommt es zur Gegenbewegung.

Dennoch ist der Wille zur Gestaltung des Zusammenlebens im Kern vorhanden und muss in der sozialen Praxis immer neu bestätigt werden, damit das Misstrauen sich nicht schließlich „durch Prozesse wechselseitiger Steigerung ins Zerstörerische wendet“ (Luhmann 2000: 100). Die Gesellschaft verfügt über Mechanismen und Institutionen, deren Aufgabe es ist, eben dies zu verhindern. Ein zentraler Mechanismus, der auch in der Debatte um das Sprachreferendum zur Anwendung gekommen ist und im Anschluss an das Referendum zur Diskurshegemonie gelangte, besteht in den „Auslegungen, welche die Durchführung von mißtrauischen Handlungen ermöglichen, aber das Mißtrauen als Einstellung wegfingieren“"(Luhmann 2000: 100). $\mathrm{Zu}$ beobachten ist dies etwa in einem Interview, das der lettische Staatspräsident Andris 
Bērziņš der österreichischen Zeitung Der Standard anlässlich des Referendums gab. Er externalisierte hier das Misstrauen gegen Teile der eigenen Bevölkerung zur Wahrung des inneren Friedens und rechnete das offensichtliche Misstrauen einem äußeren Feind zu, der Regierung Russlands, der vorgeworfen wird Lettland von innen heraus destabilisieren zu wollen:

Derzeit wird versucht, die Frage der Staatssprache zur Spaltung der Gesellschaft zu instrumentalisieren. Das ist ein gefährlicher und nicht zu tolerierender Weg, der nichts mit dem täglichen Leben der Menschen zu tun hat. [...] Die Verleugnung von Lettisch als einziger Staatssprache bedeutet auch eine Verleugnung Lettlands. Nur hier können Lettisch und die lettische Lebensart überleben. [...] Die Russen sind hier keine gefährdete Minderheit. [...] Es hat bisher keine offenen Bekundungen von Intoleranz gegeben, und ich bin sicher, dass es nach wie vor keine ernsthaften Konflikte in den Beziehungen der ethnischen Gruppen zueinander gibt. Versuche, das zu ändern, kommen nur von Leuten, die versuchen, aus einer Eskalation politisches Potenzial zu schlagen. Das ist unmoralisch und unverantwortlich. Ich bin zuversichtlich, dass die Menschen in Lettland weiser sind als die Urheber solcher politischer Initiativen.

(Stangl 2012)

Diese Aussage offenbart exemplarisch die Befürchtung der ethnisch Lettischen, dass Akteure oder Gelder aus Moskau bis heute die Politik der ehemaligen Sowjetrepubliken beeinflussen sollen. ${ }^{10}$ Neben dem hier zitierten Interview war das Referendum Anlass für zahlreiche Akteure die Einigkeit und Versöhnung zu beschwören, besonders nachdem es abschließend zu einer Ablehnung der Verfassungsänderung kam. Das Schreckgespenst einer historischen Vergangenheit, die die Gegenwart einzuholen drohte, war gebannt. Das Ringen um ein Auskommen miteinander und die Absichtsbekundungen zu einer neuen Form der Kooperation zeigt sich in der Äußerung des Präsidenten Lettlands: „Nötig sei jetzt eine ,ernsthafte Diskussion“ über das Zusammenleben in Lettland“ (Focus Online 2012).

Darüber hinaus kam es im Anschluss zu einem breiten diskursiven Konsens über die Notwendigkeit eines Miteinander:

Nach Bekanntwerden der Ergebnisse riefen sowohl lettische also auch russischsprachige Politiker zur Verständigung auf. Staatspräsident Andris Berzins sagte, nun müsse ein „Dialog“ beginnen, um „Verdächtigungen, Verstimmungen und Missverständnisse“ zu beenden. Auf der anderen Seite sagte Janis Urbanovics, der Fraktionschef des „Harmoniezentrums“, der einflussreichsten ,russischen“ Partei in Lettland, die Bürger, welche für die Zweisprachigkeit eingetreten seien, hätten sich damit nicht „gegen die Letten und ihre Sprache“ stellen wollen, sondern nur für „Dialog, wirkliche Zusammenarbeit und Integration“ gestimmt. Der russischsprachige Teil Bevölkerung habe allerdings zum Ausdruck gebracht, dass nach ihrer Meinung die bisherige „Assimilierungspolitik“ in Lettland gescheitert sei.

(Schuller 2012)

Diese Äußerungen können jedoch nicht darüber hinwegtäuschen, dass das Misstrauen als überdeckte bzw. wegfingierte Grundkonstante als vorerst ungelöster Konfliktpunkt bestehen bleibt. Schon anhand der Äußerung des lettischen Außenministers Edgars Rinkevics lässt sich

\footnotetext{
${ }^{10}$ Hier reiht sich die Meldung in der Baltischen Rundschau ein, die besagt, dass der lettische Innenminister Richards Kozlovskis die Ansicht vertritt, dass „,das im Februar 2012 durchgeführte Sprachreferendum, mit dem Russisch als Amtssprache in Lettland durchgesetzt werden sollte, unter anderem mit russischen Geld finanziert wurde. [...] Vladimir Lindermans dementierte die Behauptungen“" (Baltische Rundschau 2012).
} 
der anschließende Kurs der lettischen Regierung erahnen: „Die Regierung müsse einem bedeutenden Teil der Bevölkerung helfen, ,den lettischen Staat und seine Grundwerte' anzunehmen" (Fokus Online 2012). Es wird zwar eine Vergesellschaftung angestrebt, aber zu den Bedingungen der ethnisch lettischen. Ein echter Dialog ist nicht vorgesehen, sondern die Einheit soll von oben herab gestiftet werden, die gegenseitige symbolische Anerkennung aller Teile der Bevölkerung bleibt aus. Nur zwei Jahre nach dem Referendum, im Jahr 2014, wurde die Verfassung Lettlands um eine Präambel erweitert, die die Festschreibung der lettischen Kultur noch weiter sichert.

\section{Identität - Stereotyp - Stigma}

Die Erlebnisse der Sowjetzeit sind im kollektiven Gedächtnis der lettischen Bevölkerung noch allgegenwärtig, die Anwesenheit einer großen ethnisch russischen und russischsprachigen Bevölkerungsgruppe ist ihr Ergebnis und gleichsam besonders für die ethnischen Letten die konstante Erinnerung an das Erlittene. Es wird den ethnischen Russen und ihren Nachgeborenen eine kollektive Identität sowie Loyalitäten zum russischen Staat unterstellt.

Eine Annäherung an den Begriff Identität kann an dieser Stelle nur skizzenhaft erfolgen. Bereits Hermann Lübbe verweist auf die Zusammenhänge zwischen Geschichte bzw. individuellen Geschichten und Identität indem er konstatiert:

Die Identität von Subjekten läßt sich [...] vollständig nur über deren Geschichten vergegenwärtigen, weil diese Identität in ihrer synchronen Präsenz stets mehr enthält als das, was aus gegenwärtigen Bedingungen verständlich gemacht werden könnte.

(Lübbe 1979: 280)

Daraus schließt er, das Subjekt sei ,im Verhältnis zu der Geschichte, durch die es seine Identität hat, auch nicht deren Handlungssubjekt, sondern lediglich das Referenzsubjekt der Erzählung dieser Geschichte“ (Lübbe 1979: 280). In der vorliegenden Untersuchung geht es jedoch nicht um die Identität des einzelnen Subjekts, sondern es geht um kollektive Identität bzw. die Unterstellung einer solchen. Dies führt in den Bereich der nationalen und kulturellen Gruppenidentitäten, für die jedoch Lübbes Hinweis auf die Geschichte ebenfalls relevant ist. Georgeta Vancea zieht eine Parallele, die in der sinnstiftenden Kontinuität wurzelt:

sowohl die Identität der Person wie auch die der Nation [wird] durch narrative Sinnstiftung erzeugt, indem eine stimmige Geschichte mit Kontinuität und Sinn vor Augen geführt und den realen historischen Ereignissen die formale Kohärenz von Geschichten auferlegt wird.

(Vancea 2008: 14)

Ein wesentlicher Faktor hierbei ist das so genannte „kulturelle Gedächtnis“, das für die Gruppe die Funktion hat „Identität zu bewahren und zu reproduzieren“ (Vancea 2008: 14) und eben auch die Sprache, besonders auch Texte, die ,durch kulturelle Formung [...] und institutionalisierte Kommunikation Erinnerungen wach halten“ (Vancea 2008: 10). Auch Weichlein verweist darauf, dass schon bei Herder „Kollektive durch ihre Sprache konstituiert“ wurden und somit „ihre unverwechselbare Individualität“ generierten. Somit erzeuge die Sprache „,nicht nur individuelle Identität, sondern das typische Gepräge einer Gemeinschaft“ (Weichlein 2010: 12). Die Sinnstiftung funktioniert nach Innen zur Stärkung der eigenen Gruppenidentität(en) und selbstverständlich auch nach außen zur Abgrenzung gegen andere Grup-

ISSN 1615-3014 
pen. Der Zusammenhang mit sowie der fließende Übergang zur Genese von Stereotypen liegt hierbei auf der Hand.

Den Begriff Stereotyp ${ }^{11}$ klar zu definieren ist - trotz zahlreicher Versuche in der Forschung kompliziert und führt zu vielfältigen Nuancen. Einen allgemein gehaltenen Grundkonsens über die Merkmale von Stereotypen formuliert Groth (2003: 22):

Allgemein scheint ein Konsens darüber zu bestehen, daß Stereotype kognitive Konzepte sind, die Verallgemeinerungen über Personen oder Menschengruppen darstellen. Damit können die eigene Person oder Gruppe (Autostereotype) und auch fremde Personen oder Gruppen gemeint sein (Heterostereotype). Tajfel (1982) betont außerdem, daß Stereotype sozial geteilte Überzeugungen darstellen. (Tajfel 1982, 39) Stereotype können negativ, neutral oder auch positiv sein, im Gegensatz zu Vorurteilen, denen ausschließlich eine negative Komponente zugeschrieben wird.

Zur näheren semantischen Bestimmung wird versucht den Begriff Stereotyp von anderen Begriffen mit semantischer Schnittmenge abzugrenzen, so etwa Klischee oder Exotismus (cf. Groth 2003: 23). Besonders häufig ist die Abgrenzung zum Begriff Vorurteil. Diese ist umstritten und uneindeutig, die beiden Begriffe werden häufig synonym verwandt (cf. Groth 2003: 22). Sibylle Groth vergleicht das Verhältnis der beiden Begriffe unter Rückgriff auf Stroebe/Insko (1989) mit ,der Differenzierung gleich, die hauptsächlich zwischen Meinungen oder Einstellungen und Haltungen gemacht wird“, wonach das Stereotyp eine „Vorstellung von Merkmalen einer sozialen Gruppe oder ihrer Mitglieder“ darstellt, das Vorurteil aber ,eine negative Haltung gegenüber einer Gruppe" (Groth 2003: 22) ${ }^{12}$. Der Aspekt der Abwertung als zentrales Unterscheidungskriterium zwischen Stereotyp und Vorurteil findet sich auch bei Florack (2007: 35):

Unter dem Einfluß der ,Social-Cognition'-Forschung beziehen heutzutage die meisten Experten den „Begriff des Stereotyps“ auf „kognitive Prozesse der Unterscheidung und Verallgemeinerung“, „Vorurteil“" bezeichnet dagegen ,affektive Prozesse der Abwertung“, also die Ebene negativer Einstellungen bzw. Antipathien. Dabei darf deren Bezug zu diskriminierendem Handeln keineswegs eindeutig als Ursache-Wirkung-Relation verstanden werden, sondern muß, abhängig von Personen und gesellschaftlichem Kontext, multifaktoriell erklärt werden.

Für die vorliegende Untersuchung ist die Unterscheidung von Stereotypen und Vorurteilen jedoch nicht von zentraler Bedeutung, denn der Diskurs um das Referendum ist - bis auf die Kooperationsbeteuerungen in der Schlussphase - von abwertender Rhetorik geprägt, positive oder neutrale Stereotype werden hier nicht diskursrelevant. Somit wäre eigentlich durchge-

\footnotetext{
${ }^{11}$ Zum Ursprung der metaphorischen Übertragung des Begriffs Stereotyp aus dem Druck in einen sozialen Kontext schreibt Groth: „Ursprünglich eine bestimmte Drucktechnik bezeichnend, verwandte erstmalig der amerikanische Journalist Walter Lippmann in seiner 1922 erschienenen Monographie, The public opinion ' das Wort Stereotyp in einem sozialwissenschaftlichen Zusammenhang, um damit die fiktiven ,Bilder in unseren Köpfen“ von sozialen Gruppen (Lippmann 1964) zu umschreiben. Er umreißt mit dem Begriff Stereotyp ein Geflecht von vorgefassten Meinungen, Einstellungen und Überzeugungen, das die Wahrnehmungen des Individuums zu Strukturieren und zu steuern vermag [...].“ (Groth 2003: 20).

${ }^{12}$ Ergänzend formuliert Groth den Unterschied zwischen Stereotypen und Vorurteilen an späterer Stelle folgendermaßen: „Zusammenfassend läßt sich sagen, daß Stereotype schematisierte, sozial geteilte Selbst- und Fremdbilder sind, die Einzelpersonen oder Gruppen bestimmte Eigenschaften und Verhaltensmerkmale zu- oder absprechen. Im Gegensatz zu Vorurteilen sind ihnen nicht bestimmte Haltungen impliziert. Vorurteile aber kann man definieren als ,...affektive, emotional geladene, meist schon früh erworbene bzw. unkritisch übernommene, verhaltensrelevante Einstellungen'." (Groth 2003: 22-23).
} 
hend von Vorurteilen zu sprechen. Allerdings scheint im Sinne einer sachlichen Analyse der Terminus Stereotyp angemessener und wird daher, um keine falsche Differenzierung zu suggerieren, hier durchgehend verwendet.

Stereotype richten sich auf eine Gruppe eigener Zugehörigkeit oder eine Fremdgruppe. Sie sind ein wesentlicher Bestandteil von Gruppendynamik und Gruppeninteraktion und können sich auf verschiedenste Arten von Gruppen beziehen. Für den Diskurs um das Sprachreferendum stehen die nationalen Stereotype im Vordergrund, die in ihrer Bezogenheit auf das Abstraktum der Nation spezifische Eigenheiten aufweisen und daher kurz gesondert dargestellt werden sollen.

Nach einer wiederum allgemeinen Definition von Dabrowska gehören nationale Stereotype „,zum festen Bestandteil des Wertesystems jeder kulturellen Gruppe und werden ,Generationen hindurch als Komponenten des gesammelten Wissens der Gesellschaft übermittelt'.“ (1999: 20). ${ }^{13}$ Ein weiteres Merkmal ist die ,tendenziöse Interpretation der Tatsachen [...], die ins eigene Bild passen“ und die Neigung ,die existierenden Grundvorstellungen für fundiertes Wissen“ (Dabrowska 1999: 21) zu halten. Während sich Dabrowska bei ihren Ausführungen wesentlich auf Heterostereotype konzentriert, die das Verhältnis zwischen Nationen betreffen, richtet Hroch seine Aufmerksamkeit auf nationale Autostereotype und ihre Funktionen: „Abstrakte Ideen und komplexe Symbole konnten zum Ausgangspunkt nationaler Imagination gerade deshalb werden, weil sie sich zu Stereotypen entwickelten“. Dabei sind wesentliche Merkmale von Stereotypen nach seiner Auffassung die Abschottung gegen Überprüfbarkeit und „unkritische Verallgemeinerungen“ (Hroch 2005: 209).

Die Stereotype können ,beschrieben werden, als ,vereinfachende und generalisierende Urteile mit emotional wertender Tendenz (Uta Quasthoff)“ oder aber ,als ,verfestigte Überzeugungen, Alltagskategorisierungen, Typisierungen der Umwelt`, die sich auf die Realität beziehen und Einfluss auf sie ausüben (Klaus Roth)“ (Hroch 2005: 209). Dabei sind sie zu sehen als Instrumente, durch welche ,die Gruppenangehörigen ein System von Vorstellungen von sich selber schaffen, aufrechterhalten und so die Einordnung der Einzelnen mit Hilfe bestimmter vorgegebener Indikatoren“ durchführen, mit dem Ziel „,der Unterscheidung zwischen dem ,WIR` und dem ,SIE‘ Ausdruck zu verleihen“ (Hroch 2005: 209). Sie haben eine Orientierungsfunktion für den Einzelnen, indem Sie - wie auch schon in Bezug auf das Misstrauen beschrieben - Komplexität reduzieren und die „Annahme der neu angebotenen Identifikation mit der Nation“ (Hroch 2005: 209-210) erleichtern. Die Stereotype werden, wie andere Vorstellungen einer Gesellschaft, über das ,Kollektivgedächtnis‘ weitergegeben (cf. Hroch 2005: 210), wobei Hroch die Wandelbarkeit der Stereotype explizit unterstreicht (2005: 210):

Es gehört zu den unreflektierten Annahmen der Forschung, nationale Stereotype als etwas Stabiles und Abgeschlossenes anzusehen, als Einstellungen, die automatisch und von Anfang an von allen Angehörigen der nationalen Gemeinschaft angenommen wurden. Man muss jedoch immer das soziale und kulturelle Milieu, in dem das Stereotyp entstand, von seinem weiteren Geschick unterscheiden, davon, in welchem Maße es von den Angehörigen der nationalen Gemeinschaft angenommen wurde - oder nicht.

\footnotetext{
${ }^{13}$ Dabrowa diskutiert nachfolgend den Begriff Nationalcharakter, auf den allerdings an dieser Stelle nicht auch noch eingegangen werden soll. Allerdings postuliert sie die Notwendigkeit eines Konzepts von Nationalcharakter als Voraussetzung für die Genese von Stereotypen und Vorurteilen.
} 
In der Debatte um das Referendum offenbaren sich die im Misstrauen begründeten und von den Angehörigen der nationalen Gemeinschaft angenommen ,affektiven Prozesse der Abwertung", besonders in der Perspektive der ethnisch lettischen auf die ethnisch russischen, wie sich an einem Zitat der umstrittenen Schriftstellerin Māra Zālīte, verdeutlichen lässt. In ihren Äußerungen und besonders in ihrer Vehemenz und Polemik wird Zālīte zwar in der lettischen Öffentlichkeit durchaus kritisch wahrgenommen, dennoch formuliert sie beispielhaft den Kern der auf die Spitze getriebenen lettischen Perspektive auf den ethnisch russischen Bevölkerungsteil. Ein Beitrag Zālītes vom 29.01.2012 wurde im Rahmen der Berichterstattung für Deutschland folgendermaßen ins Deutsche übersetzt:

\begin{abstract}
„Der russische Sprachraum, dank den Mitteln der Massenmedien, vereint sich freizügig von Ventspils bis Wladiwostok. Für die russische Sprache bestehen die allerbesten Zukunftsperspektiven. Für die hundertfach kleinere lettische Sprache gibt es solch große Sicherheiten nicht. Unsere althergebrachte baltische Sprache (In der Welt gibt es davon nur noch zwei VariantenLettisch und Litauisch) enthalten die von der UNESCO geschützten Daina-Volkslieder als klassische Literatur. Das ist der Grundstein unseres Staates und des Bestehens unserer Nation. Ich schäme mich, dass man wieder und wieder solch selbstverständlichen Dinge wiederholen muss. Es wäre nur natürlich, wenn die eigene Staatssprache von allen Bürgern geehrt und geachtet würde, unterstützt und vertreten von jeder ethnischen Gruppe. " [...] So ist Zālītes rhetorische Frage verständlich: „,Ob tatsächlich des linguistischen Komforts halber Lettlands Russen nicht verhindern wollen, die lettische Sprache der Vernichtung preiszugeben?“ [...] „Wie können Lettlands Russen erlauben, dass sie sich von einem Vladimirs Iljičs manipulieren lassen, welcher sich als Nationalbolschewist ausgibt? Um es in eine für Europa verständliche Terminologie zu übersetzen, diese Ideologie nennt man Neonazismus. Der ist verbrecherisch und in demokratischen Staaten verboten. “
\end{abstract}

(Hervorhebungen im Original; Bongartz 2012)

Die Äußerungen Zālītes zeigen folgende markante Punkte:

(a) das Postulat einer natürlichen und daher unhinterfragbaren Ordnung mit normativem Charakter (,Es wäre nur natürlich, wenn die eigene Staatssprache von allen Bürgern geehrt und geachtet würde, unterstützt und vertreten von jeder ethnischen Gruppe."), weiterhin

(b) die Bezugnahme auf eine Gruppe als DER kollektive Russe, enthalten in der Formulierung „Lettlands Russen“ sowie

(c) die Unterstellung von und Bezugnahme auf unterstellte charakterliche Defizite wie Egoismus und Bequemlichkeit, kulturelle Ignoranz, Illoyalität den echten Letten gegenüber, leichte Manipulierbarkeit und politische Ahnungslosigkeit. Hiermit ist die letzte Auffälligkeit aufs engste verknüpft, nämlich

(d) die implizierte moralische Minderwertigkeit, die eine gesellschaftliche Ausgrenzung und politische Nicht-Beachtung legitimiert, um die postulierte natürliche Ordnung zu schützen.

Im Zentrum der Äußerung steht als Konsequenz des Misstrauens strukturell die Annahme der Unzuverlässigkeit des Russen, der den Letten bei der nächsten Gelegenheit hintergehen und den lettischen Staat an Russland verraten würde. Die Individualität einzelner Akteure oder die 
Diversität der ethnisch russischen insgesamt wird völlig negiert und den ebenfalls illegitim vereinheitlichten ethnisch lettischen polar entgegen gesetzt. Die postulierte Ordnung kann für Lettland nicht aus der historischen Erfahrung abgeleitet und als goldenes Zeitalter heraufbeschworen werden, daher muss die angestrebte Normvorstellung als naturgegeben deklariert werden, um sie durch die Meta-Ebene der Hinterfragbarkeit zu entziehen. Der kollektive Russe als Störer der natürlichen Ordnung erscheint hier als zu überwindendes Hindernis bei der durch Naturgesetz legitimierten Realisierung der Utopie. Die charakterlichen Defizite und die daraus resultierende moralische Minderwertigkeit korrespondiert hierbei mit der Opposition des Russen zur natürlichen Ordnung, was ihn zum Außenseiter per se macht.

Von diesem Zentrum leiten sich die beiden wesentlichen Spielarten der Stereotype über den Russen ab, die im Zitat Zālītes zwar gekoppelt auftreten, aber analytisch getrennt werden müssen. Zum einen das Stereotyp des bösen Russen, zum anderen das Stereotyp des dummen Russen. Das Stereotyp des bösen Russen geht von der bewussten, böswilligen Unzuverlässigkeit, also Hinterhältigkeit, aus. Hier ist der Russe der Artfremde, der außerhalb der Gemeinschaft der moralisch-zivilisierten Menschen Befindliche, der Spion, die 5. Kolonne, der Verräter, der als Handlanger und Gefolgsmann der russischen Regierung eine Gefahr für Lettland darstellt, weil er Harmlosigkeit vortäuscht und nur darauf wartet den Letten ans Messer liefern zu können. Das Stereotyp des dummen Russen geht von der politischen Ahnungslosigkeit und Manipulierbarkeit, also einer grundsätzlichen intellektuellen Unterlegenheit des Russen aus, der zwar keine eigenen negativen Absichten hegt, aber von außen (der russischen Regierung) jederzeit gegen Lettland eingesetzt werden kann, ohne dass er selbst es begreifen könnte. Die Äußerung Zālìtes ist gespickt mit Hinweisen auf das intellektuelle Defizit, oder aber die Denkfaulheit, die dem Erkennen und Befolgen der postulierten natürlichen Ordnung entgegensteht. Es kommt oft zur Kopplung der beiden strukturell gegensätzlichen Varianten, sie sind etwa impliziert in Zālītes Frage „Wie können Lettlands Russen erlauben, dass sie sich von einem Vladimirs Iljičs manipulieren lassen, welcher sich als Nationalbolschewist ausgibt?" Der umstrittene Status Vladimir Lindermans und seine Verbindung zum Nationalbolschewismus geben Zālīte Anlass einerseits von Manipulation zu sprechen, was explizit auf den dummen Russen verweist, gleichzeitig unterstellt sie, dass es eine zugelassene, also bewusst hingenommene Manipulation ist, was wiederum auf den bösen Russen verweist. Die Eigenschaften, die dem Russen zugeschrieben werden, suggerieren, wie man bei Florack findet, „eine Art von Wesenhaftigkeit (,essence“)“ (Florack 2007: 36). Diese explizite Diagnose zum Wesen des Russen, schließt aber auch eine implizite Vorstellung des Letten und seines Wesens mit ein, denn das lettische Autostereotyp ist dem russischen Heterostereotyp (cf. Hahn/Hahn 2002: 27) diametral entgegengesetzt. Das Bedürfnis einer Abgrenzung der eigenen Identität wird hier auf die Spitze getrieben, indem Māra Zālīte stellvertretend für die Letten implizit nicht nur als kämpferische Verteidigerin des Lettentums auftritt, sondern auch für sich in Anspruch nimmt, die natürliche Ordnung durchschaut zu haben und ihr zu ihrem Recht verhelfen zu wollen. Vergegenwärtigt man sich die Spielarten des dummen Russen und des bösen Russen wird deutlich, dass das lettische Autostereotyp die Kehrseite abbildet, also den schlauen Letten und den guten Letten. Der schlaue Lette erkennt nicht nur das Komplott gegen Lettland, sondern auch die Manipulation, die dem dummen Russen widerfahren ist. Der gute Lette verteidigt aus dieser Erkenntnis heraus nicht nur das Lettentum, also den Kern der eigenen Identität, sondern er leistet auch durch die Herstellung oder Aufrechterhaltung der

ISSN 1615-3014 
natürlichen Ordnung einen Dienst an der zivilisierten Weltgemeinschaft. Somit kann er die moralische Überlegenheit in Anspruch nehmen. Der Topos des Durchschauens eines Komplotts, der auch im Interview mit dem Präsidenten deutlich wurde, impliziert die Anerkennung einer materiellen, personalen und finanziellen Übermacht von außen, bei gleichzeitiger intellektueller und moralischer Überlegenheit und Zähigkeit des Letten.

Alltägliche Stereotype zwischen ethnisch lettischen und ethnisch russischen Gruppen, etwa über Lebensstil oder Kultur, sportliche Interessen oder Mode, sind ebenso zahlreich aufzufinden, da die beiden Bevölkerungsteile im privaten Bereich die Tendenz haben unter sich zu bleiben. Im lettischen Kollektivgedächtnis und in der medialen Öffentlichkeit spielen diese Stereotype jedoch eine untergeordnete Rolle, da die negativen Stereotype über eine existentielle Bedrohung durch den Russen eine weit höhere emotionale Affizierung bewirken, denn sie reaktivieren immer wieder aufs Neue die historisch bedingten Ängste der ethnisch lettischen Gruppen vor Unterdrückung. Inwiefern es aufgrund dieser Deutungsmuster zu diskriminierenden Handlungen kommt, gehört zu den alltäglichen politischen Streitthemen Lettlands.

Ein Autostereotyp, das jedoch von beiden Gruppe gleichsam für sich in Anspruch genommen wird, betrifft die Selbstwahrnehmung in der Rolle der Opfer der jeweils anderen Gruppe im Kontext der jeweiligen historischen Umstände: So sehen sich die ethnisch lettischen Gruppen als Opfer der Sowjetpolitik und ihrer Folgen bis in die Gegenwart. „Lettland möchte insbesondere die Opfer der sowjetischen Okkupation anerkannt wissen. Das Narrativ von der Befreiung von der sowjetischen Okkupation ist deshalb für die Konstruktion des lettischen Selbstbildes von besonderer Bedeutung“" (Wezel 2008: 213). Die ethnisch russischen Gruppen sehen sich seit der zweiten Unabhängigkeit in den 90er Jahren als Opfer der lettischen Politik, wobei sich durchaus auch ethnisch russische Gruppen als Opfer der Sowjetpolitik der Zwangsumsiedlungen wahrnehmen. In diesem Sinne handelt es sich um eine doppelte Opferrolle der ethnisch russischen Gruppen: zuerst von der Sowjetregierung in Lettland angesiedelt und dann von der lettischen Regierung diskriminiert. So gewinnt der außenstehende Beobachter den Eindruck, dass es in der lettischen Bevölkerung im Autostereotyp nur Opfer und im Heterostereotyp nur Täter gibt. Der Oper-Topos findet sich implizit bei Zālīte, indem sie auf die quantitative Überlegenheit der russischen Sprache und Kultur verweist (,Der russische Sprachraum, dank den Mitteln der Massenmedien, vereint sich freizügig von Ventspils bis Wladiwostok.") und daraufhin die lettische Sprache als quantitativ unterlegen und von Auslöschung bedroht charakterisiert. „The pressure to use the heritage language can be particularly strong where the language tied to an ethnic identity is perceived as threatened." (Fought 2006: 29) Dieses Bedrohtsein der Sprache - denn der Anlass ist ja das Sprachreferendum wird zu einer Bedrohung des Lettentums generalisiert und impliziert damit ein Bedrohtsein der lettischen Identität an sich, die in der zweiten Hälfte des 20. Jahrhunderts bereits Opfer der russischen Willkür gewesen ist und vor neueren Angriffen geschützt werden muss. Zur Rettung der „heritage language“ Lettisch, soll diese nicht nur von den ethnisch lettischen Gruppen gesprochen, sondern muss auch von den ethnisch russischen Gruppen übernommen werden. Eine Thematisierung der existenziellen Bedrohung findet sich im zuvor genannten Zitat des Präsidenten Lettlands (,Die Verleugnung von Lettisch als einziger Staatssprache bedeutet auch eine Verleugnung Lettlands. Nur hier können Lettisch und die lettische Lebensart überleben."), wobei die Opferrolle aus der historischen Erfahrung impliziert wird. Gleichsam wird im direkten Gegenzug den ethnisch russischen Gruppen die Opferrolle explizit ab-

ISSN 1615-3014 
erkannt („Die Russen sind hier keine gefährdete Minderheit ${ }^{(*)}$. Bemerkenswert ist, dass die Begründung des Autostereotyps Opfer sich jeweils aus unterschiedlichen Wahrnehmungsmustern ergibt. Während für die ethnisch lettischen Gruppen die auf vielerlei Ebenen (materiell, finanziell, Bevölkerung) quantitative Unterlegenheit gegenüber Russland, der russischen Regierung und teils auch der ethnisch Russischen im eigenen Lande ausschlaggebend ist, sehen sich die ethnisch russischen Gruppen in Lettland, da sie der Größe Russlands gewiss sein können, als qualitativ benachteiligt, etwa in der Gesetzgebung. Doch linguistische Untersuchungen, die die lettische Germanistin Jūlija Zālìte im Rahmen der eingangs erwähnten Tagung „Verfall der Sitten und Moral“ - Diskursanalysen der Ablehnung/Abgrenzung gesellschaftlicher Wertvorstellungen vorstellte, belegen, dass die öffentliche Debatte um das Referendum auf Seite der ethnisch lettischen Einwohner sehr viel heftiger ausfällt als auf Seite der ethnisch russischen Einwohner. Der existentielle Charakter des Themas aus lettischer Perspektive sowie die unverarbeitete Sowjeterfahrung sind genannt worden. Hinzu kommt die Instabilität der eigenen Position in der Gegenwart. Die Republik Lettland kämpft mit wirtschaftlichen Problemen und Auswanderung signifikant großer Bevölkerungsteile. Die Eigenstaatlichkeit hat lediglich eine fast 100 Jahre währende Tradition, die durch die Sowjetzeit (1945-1991) für 46 Jahre unterbrochen war. Die ältere Geschichte Lettlands ist bestimmt von Fremdherrschaft, die Gegenwart wird überschattet von einer geographischen Mittellage zwischen West und Ost.

In gleichem Maße wie es bei der Diskussion um das Referendum um die Abwehr der Zerstörung durch den Russen ging, ging es auch um die Bejahung und Selbstvergewisserung des Eigenen. Die Gemeinschaft der Letten manifestiert darüber hinaus auch in der Formulierung der Präambel von 2014 nicht nur implizit die Abgrenzung, sondern auch offensiv die Festigkeit des Eigenen durch Fest-Schreibung im wörtlichen Sinn. Nationale Stereotype dienen, wie bereits dargelegt wurde, nicht unwesentlich dazu ,nach innen zu integrieren und nach außen abzugrenzen“ (Schönwald 2012: 78). Das Problematische für Lettland an dieser Dynamik ist die Tatsache, dass sich beide Gruppen, die einander nicht nur als getrennte Gruppen sondern als getrennte nationale bzw. ethnische Gruppen begreifen, denselben Staat bewohnen und derselben Staatsordnung unterliegen. Es treffen daher nationale Stereotype innerhalb eines Staates aufeinander und es ist an dieser Stelle nicht zu klären, ob sich ein Teil der Stereotype vielleicht erst durch dieses Zusammenleben entwickeln konnte. Legt man Schönwalds Beobachtung zugrunde, dass die Ingroup ,dabei stets höherwertig dargestellt [wird] als die ,Anderen, bzw. die Outgroup. “" (Schönwald 2012: 78) und berücksichtigt man auch, dass beide Gruppen sich als Opfer der jeweils anderen Gruppe wähnen, erschließt sich die Starrheit der Positionen sowie die Tendenz zur politischen Spaltung.

Die Alltagspraxis, in der die Bevölkerungsteile im Umgang miteinander Erfahrungen sammeln, führt langsam zu Relativierungen der Stereotype, die durch das grundlegende Misstrauen doch eine Resistenz gegen versöhnende Alltagsbeispiele aufweist. Zudem lässt sich eine Tendenz zum „subtyping“ (Schönwald 2012: 80) beobachten. Der einzeln durchaus in Erscheinung tretende - als lettischer Sicht vernünftige - Russe wird als ein aus der Art geschlagenes Exemplar einer ansonsten homogenen Gruppe eingestuft, sogar wenn sich solche Beobachtungen häufen. Verstärkend wirkt hier möglicherweise der Linguistic Intergroup Bias. „Dieser besagt, dass positives Verhalten der Eigengruppe eher abstrakt, negatives häufiger konkret beschrieben wird. Für die Beschreibung von Verhalten der Fremdgruppe gilt das Ge-

ISSN 1615-3014 
genteil“" (Schönwald 2012: 80). Für die Beurteilung einer Gruppe spielt jedoch die abstrakte Zuschreibung eine größere Rolle, weil sie als allgemeingültig und somit übergeordnet angesehen wird. Der vernünftige Russe wird somit zum immer wieder vorkommenden Einzelfall, nicht zur Regel. Die Zuordnung zum Sub-Typ vernünftiger Russe orientiert sich wesentlich über die Sprachfähigkeit. Lettisch-sprechende Russen werden tendenziell akzeptiert, Russen, die die Sprache nicht beherrschen, werden in besonderem Maße als verdächtiger Fremdkörper wahrgenommen, woran die Bedeutsamkeit des Sprachreferendums sich klar offenbart.

Die bisherigen Überlegungen zeigen eine komplexe Situation wechselseitigen Misstrauens, in welcher der ethnisch lettische Bevölkerungsteil über die politische Definitionsmacht verfügt, wobei sich beide Bevölkerungsgruppen als Opfer der jeweils anderen wahrnehmen.

Bei genauerer Betrachtung der Perspektive der ethnisch lettischen Bevölkerung, die sich im Referendum lediglich gebündelt zeigt, scheint es lohnend über den Stereotypenbegriff hinauszugehen. Erving Goffman beschreibt in seiner Schrift Stigma 1963 (deutsche Übersetzung 1967) die Funktionsweisen gesellschaftlicher Stigmatisierung. Die Stigmatisierung, entkoppelt vom Bezug auf ein körperliches Gebrechen oder eine visuelle oder medizinische Anomalie, kann verstanden werden als eine Eigenschaft oder ein Set von Eigenschaften, die, wenn sie erkannt werden, als Ausweis der Unehre dienen, und den so Stigmatisierten gesellschaftlich abwerten. Die Konsequenz ist, dass er aus der angenommenen Gruppe der gesellschaftlich Normalen also jener Personen, die keine Träger dieses Stigmas sind, ausgegrenzt wird. Im alltäglichen Umgang erschafft eine Gesellschaft

die Mittel zur Kategorisierung von Personen und den kompletten Satz von Attributen, die man für die Mitglieder jeder dieser Kategorien als gewöhnlich und natürlich empfindet. Die sozialen Einrichtungen etablieren die Personenkategorien, die man dort vermutlich antreffen wird.

(Goffman 1975: 9)

Ohne weitere Reflexion sind diese Kategorien Grundlage des alltäglichen Sozialverhaltens und den normativen Erwartungen daran. Den Prozess in welchem die normale Person in den Wahrnehmungen der Umwelt eben den Status des Normalen verliert, beschreibt Goffman (1975: 10) wie folgt:

Während der Fremde vor uns anwesend ist, kann es evident werden, daß er eine Eigenschaft besitzt, die ihn von anderen in der Personenkategorie, die für ihn zur Verfügung steht, unterscheidet; und diese Eigenschaft kann von weniger wünschenswerter Art sein [...]. In unserer Vorstellung wird sie so von einer ganzen und gewöhnlichen Person zu einer befleckten, beeinträchtigten herabgemindert. Ein solches Attribut ist ein Stigma, besonders dann, wenn seine diskreditierende Wirkung sehr extensiv ist; manchmal wird es auch ein Fehler genannt, eine Unzulänglichkeit, ein Handykap. Es konstituiert eine besondere Diskrepanz zwischen virtualer und aktualer sozialer Identität.

Die aktuale soziale Identität umfasst die Eigenschaften, die einem Individuum oder einer Gruppe tatsächlich nachgewiesen werden können. Die virtuale soziale Identität hingegen beruht auf Zuschreibungen und Forderungen, es ist eine „Charakterisierung ,im Effekt"“ (Goffman 1975: 10). Diese manifestiert sich im öffentlichen Diskurs. Es werden Zuschreibungen gemacht, die als Handykap angesehen werden: die Abweichung von der (Sprach-)Norm, die Unterstellung den Staat zersetzen zu wollen, durch die Verweigerung der so dargestellten lettischen Tugenden, denen die Sprache offiziell beigerechnet wird. Hier zeigt sich die Spra- 
che als akustisches Stigmasymbol, also als Zeichen, das auf das prestigemindernde Handykap des Russischsein verweist (cf. Goffman 1975: 59). Die kollektive Identität soll eine rein lettische sein, ist aber faktisch eine vielschichtig lettisch und russisch durchsetzte und vermischte Identität, die sich beim Individuum in den verschiedensten Formen und Schattierungen zeigt. Das Referendum durchbricht die verordnete Normalität und ist daher für die Gegner des Referendums eine unerhörte Provokation und Ausweis eines kollektiven Stigmas in Form der erwarteten und nunmehr wieder zu Schau gestellten Unehrenhaftigkeit der ohnehin ausgegrenzten Russischsprecher. Das Urteil über die Russen fällt in vielen öffentlichen Redebeiträgen mit Blick auf die virtuale kollektive Identität und nicht mit Blick auf die aktualen individuellen Identitäten der betroffenen Personen. Der Versuch einer Normalisierung durch gesetzliche Normierung muss scheitern.

\section{$5 \quad$ Fazit}

Die nicht verarbeiteten historischen Umstände und Verlusterfahrungen seit der zweiten Hälfte des 20. Jahrhunderts führten dazu, dass sich zwei große ethnische Gruppen in Lettland voneinander abgrenzen. Während sich ein grundsätzliches Misstrauen der ethnisch russischen Bevölkerung auf Fragen des sozialen Miteinanders bezieht und auf die empfundene Ablehnung und Abwertung, ist das Misstrauen der ethnisch lettischen Bevölkerung an existenzielle Fragen der eigenen Identität, wie Staatlichkeit, Sprache und Kultur geknüpft. Jede Gruppe sieht sich im Autostereotyp als Opfer der jeweils anderen Gruppe. Kernpunkt des Konflikts ist die Frage nach den Normen des Gemeinwesens und wer diese wie festlegen darf. Die aktuelle Normierung in Frage zu stellen erhält im Diskurs der ethnisch lettischen Gruppen den Status einer existentiellen Bedrohung von Identität, Sprache, Kultur und Eigenstaatlichkeit, weil eine Rückkehr zur russisch-dominierten Normierung befürchtet wird. Ereignisse wie das Sprachreferendum reaktivieren diese Befürchtungen. Das Resultat ist eine nochmalige Steigerung der Normierung durch die ethnisch lettischen Gruppen, wie die 2014 verabschiedete Präambel der Verfassung zeigt. Das historisch begründete Misstrauen, das durch anhaltende negative Stereotypisierung, die sich in ihrer Langlebigkeit und Intensität auch als Stigmata beschreiben ließe, aufrechterhalten wird, erweist sich als zählebig, muss aber gleichsam für ein funktionierendes, stabiles Gemeinwesen überwunden werden. Eine wechselseitige symbolische Anerkennung wäre ein erster Schritt zur Annäherung. Das Verständnis für die andere Gruppe und das Abbauen des Misstrauens und der Stereotype kann aber lediglich über eine alltägliche Vergesellschaftung und das Ende gegenseitiger Abgrenzung gelingen, da Ressentiments über die Generationen weitergegeben werden.

Die geplante Vereinheitlichung des Schulsystems und die Aufhebung der Trennung in lettische und russische Schulen könnte ein wichtiger Schritt sein, die Staatssprache zu einem grundlegenden Instrument zu machen, auf das alle Einwohner natürlicherweise auf gleichem Niveau zurückgreifen können. Russisch sollte dabei flächendeckend als zusätzliche Sprache zur Auswahl stehen, wie es vielfach auch praktiziert wird, denn ein Verständnis für den praktischen Nutzen der russischen Sprache etwa in den lettischen Außenhandelsbeziehungen (unterbrochen von den derzeitigen Wirtschaftssanktionen zwischen der Europäischen Union und Russland) ist weit verbreitet. 
Punktuell leistet die Populärkultur Anknüpfungspunkte für die jüngere Generation beider Gruppen. Gerade für die jüngere Generation, die keine persönlichen Erinnerungen mehr mit der Sowjetunion verbindet, hat das Konzept eines offenen Europas und einer europäischen Identität eine hohe Attraktivität. Lettland verfügt über eine hohe Akademikerquote und viele Studierende lernen über Auslandssemester oder sonstige Auslandsaufenthalte andere Kulturen kennen. Dies erlaubt jenen Personen die Annahme einer europäischen Identität, die von den ethnisch-sprachlichen Querelen Lettlands ermüdet sind.

\section{Literatur}

CVL = Central Election Commission of Latvia (2012): Referendum on the Draft Law "Amendments to the Constitution of the Republic of Latvia". http://cvk.lv/pub/public/30287.html [20.02.2016].

Bongartz, Udo (2011a): „Lettland - Verfassungsreferendum für die Staatssprache Russisch spaltet die öffentliche Meinung“. Lettische Presseschau, 11.11.2011. www.lettischepresseschau.de/politik/lettland/489-lettland-verfassungsreferendum-fuer-die-staatsspracherussisch-spaltet-die-oeffentliche-meinung [05.04.2015].

Bongartz, Udo (2011b): „Lettland: Russisch-Referendum in der zweiten Runde erfolgreich Nun wird das Parlament entscheiden“. Lettische Presseschau, 04.12.2011. www.lettischepresseschau.de/politik/lettland/494-lettland-russisch-referendum-in-der-zweiten-rundeerfolgreich [05.04.2015].

Bongartz, Udo (2011c): „Lettland: Parlamentarier lehnen das Russisch-Referendum ab“. Lettische Presseschau, 27.12.2011. www.lettische-presseschau.de/politik/lettland/498lettland-parlamentarier-lehnen-das-russisch-referendum-ab [05.04.2015].

Bongartz, Udo (2012): „Lettland: Vorläufiges Endergebnis der Volksabstimmung: Zirka 25 Prozent für und 75 Prozent gegen Russisch als zweite Staatssprache“. Lettische Presseschau, 19.02.2012. www.lettische-presseschau.de/politik/lettland/511-lettlandvorlaeufiges-endergebnis-der-volksabstimmung-cirka-25-prozent-fuer-und-75-prozentgegen-russisch-als-zweite-staatssprache [05.04.2015].

Dabrowska, Jarochna (1999): Stereotype und ihr sprachlicher Ausdruck im Polenbild der deutschen Presse. Tübingen: Narr.

Focus Online (2012): „Referendum in Lettland. Klares Nein zu Russisch“. 19.02.2012. www.focus.de/politik/weitere-meldungen/referendum-in-lettland-klares-nein-zurussisch_aid_715720.html [05.04.2015].

Fought, Carmen (2006): Language and Ethnicity. Cambridge: Cambridge University Press. Gnauck, Gerhard (2012): „Der Kampf der Russen um ihre Sprache in Lettland“. Die Welt Online. 16.02.2012. www.welt.de/politik/ausland/article13870448/Der-Kampf-derRussen-um-ihre-Sprache-in-Lettland.html [05.04.2015].

Goffman, Erving (1975): Stigma. Frankfurt a. M.: Suhrkamp.

Groth, Sibylle (2003): Bilder vom Fremden. Zur Konstruktion kultureller Stereotype im Film. Marburg: Tectum.

Hroch, Miroslav (2005): Das Europa der Nationen. Die moderne Nationsbildung im europäischen Vergleich. Göttingen: Vandenhoeck \& Ruprecht.

Kusber, Jan (2014): „Tradition und Neuanfang: Wege der Geschichte Lettlands im 20. und 21. Jahrhundert“. In: Bogojavlenksa, Svetlana/Kusber, Jan (eds.): Tradition und Neuan- 
fang. Forschungen zur Geschichte Lettlands an der Wende vom 20. zum 21. Jahrhundert. Berlin, LIT: 1-12.

Liebhart, Karin (2009): „Divergierende Erinnerungskulturen und gedächtnispolitische Konflikte als Faktoren im europäischen Integrationsprozess: Das Beispiel der Baltischen Staaten Estland und Lettland“. In: Isop, Utta/Ratkovic, Viktorija/Wintersteiner, Werner (eds.): Spielregeln der Gewalt. Kulturwissenschaftliche Beiträge zur Friedens- und Geschlechterforschung. Bielefeld, Transcript: 119-140.

LM = Lettisches Migrationsamt (2014): „Latvijas iedzivotaju sadalijums pec valstiskas piederibas“ [Einwohner-Statistiken für $\quad$ Lettland], 4 Seiten. www.pmlp.gov.lv/lv/assets/01072013/01.01.2014/ISVP_Latvija_pec_VPD.pdf [20.02.2016].

LM = Lettisches Migrationsamt (2015): „Latvijas iedzivotaju sadalijums pec nacionala sastava un valstiskas piederibas" [Einwohner-Statistiken für Lettland], 7 Seiten. www.pmlp.gov.lv/lv/assets/documents/statistika/01.01.2015/ISVN_Latvija_pec_TTB_VP D.pdf [20.02.2016].

LRS = Latvijas Republikas Satversme (s. a.): Die lettische Verfassung. http://likumi.lv/doc.php?id=57980 [20.02.2016].

Lübbe, Hermann (1979): „Zur Identitätspräsentationsfunktion der Historie“. In: Marquard, Odo/Stierle, Karlheinz (eds.): Identität. München, Fink: 277-292.

Luhmann, Niklas (2000): Vertrauen. Ein Mechanismus der Reduktion sozialer Komplexität. 4. Auflage. Stuttgart: Lucius \& Lucius.

Schönwald, Antje (2012): Identitäten und Stereotype in grenzüberschreitenden Verflechtungsräumen. Das Beispiel der Großregion. Wiesbaden: Springer.

Schuller, Konrad (2012): „Gegen Russisch als Staatssprache“. Frankfurter Allgemeine Zeitung. 19.2.2012. www.faz.net/aktuell/politik/ausland/abstimmung-in-lettland-gegenrussisch-als-staatssprache-11655243.html [05.04.2015].

Stangl, Andreas (2012): „Mit Russisch als zweiter Amtssprache würde man Lettland liquidieren“. Der Standard. 17.02.2012. http://derstandard.at/1328508068138/Mit-Russisch-alszweiter-Amtssprache-wuerde-man-Lettland-liquidieren [05.04.2015].

Vancea, Georgeta (2008): Toleranz und Konflikt. Interkulturelle Dimensionen der deutschsprachigen Gegenwartsliteratur. Heidelberg: Winter.

$\mathrm{Vvl}=$ Valsts valodas likums (s. a.): Das lettische Sprachgesetz .

http://likumi.lv/doc.php?id=14740 [20.02.2016] (deutsche Bemerkungen zum Gesetzestext mit Teilübersetzung:

www.forost.lmu.de/sprachdatenbank/sprachdatenbank.php?display=Lettisch:sprachkultur:s prachpolitik [20.02.2016]).

Weichlein, Siegfried (2012): Nationalbewegungen und Nationalismus in Europa. 2. Auflage. Darmstadt: Wissenschaftliche Buchgesellschaft.

Wezel, Katja (2008): „Nationskonstruktion und Abgrenzung. Lettland nach dem Ende der Sowjetherrschaft“. In: Fritz, Regina/Sachse, Carola/Wolfrum, Edgar (eds.): Nationen und ihre Selbstbilder. Postdiktatorische Gesellschaften in Europa. Göttingen, Wallstein: 211232. 